\title{
Erratum to: Rigidity in motivic homotopy theory
}

\author{
Oliver Röndigs · Paul Arne Østvær
}

Published online: 2 June 2011

(C) Springer-Verlag 2011

\section{Erratum to: Math Ann (2008) 341:651-675 DOI 10.1007/s00208-008-0208-5}

As pointed out to us by Markus Spitzweck the adjunction employed in the proof in the original paper in page 666 requires an evident replacement. With this modification all the results of our paper become valid.

From [2, Sect. 4], let $f: S \rightarrow R$ be a map of base schemes. Base change and pushforward along $f$ determine a Quillen adjunction between motivic symmetric spectra:

$$
f^{*}: \mathbf{M S S}_{R} \rightleftarrows \mathbf{M S S}_{S}: f_{*}
$$

When $S$ is a filtered limit of smooth schemes over $R$ with affine transition maps, Lemma 4.1 in [2] displays a Quillen adjoint pair:

$$
f_{\sharp}: \mathbf{M S S}_{S} \rightleftarrows \mathbf{M S S}_{R}: f^{*}
$$

The total derived Quillen adjunction can only exist when restricting to constructible objects in the motivic stable homotopy category $\mathbf{S H}_{S}$ of $S$, cf. [1]. But by replacing

The online version of the original article can be found under doi:10.1007/s00208-008-0208-5.

O. Röndigs ( ()

Institute of Mathematics, University of Osnabrïck, Osnabrück, Germany

e-mail: oroendig@math.uni-osnabrueck.de

P. A. Østvær

Department of Mathematics, University of Oslo, Oslo, Norway

e-mail: paularne@math.uio.no 
(2) with (1) we shall note that all the results in [2] become valid with only minor emendations.

Let $K / k$ be an extension of algebraically closed fields and denote by $f$ the induced map of affine schemes. Assume $\ell$ is relatively prime to the exponential characteristic of the base field $k$.

In the statement of [2, Theorem 4.10] we replace the counit $\epsilon: f_{\sharp} f^{*} \rightarrow$ id of (2) by the unit $\eta: \rightarrow f_{*} f^{*}$ of the adjunction (1). A corrected version of Theorem 4.10 reads: For motivic symmetric spectra $E$ and $F$ there is an isomorphism

$$
\eta(F): \operatorname{Hom}_{\mathbf{S H}(k)}\left(E, F / \ell^{v}\right) \stackrel{\cong}{\rightarrow} \operatorname{Hom}_{\mathbf{S H}(k)}\left(E, f_{*} f^{*} F / \ell^{v}\right) .
$$

Via the adjunction (1) the target of the isomorphism identifies with $\operatorname{Hom}_{\mathbf{S H}(K)}\left(f^{*} E\right.$, $\left.f^{*} F / \ell^{\nu}\right)$. Moreover, for the motivic sphere spectrum $\mathbf{1}_{k}$ there is an isomorphism

$$
\operatorname{Hom}_{\mathbf{S H}(K)}\left(f^{*} \mathbf{1}_{k}, f^{*} F / \ell^{v}\right) \cong \underset{k \subset R \subset K}{\operatorname{colim}} \operatorname{Hom}_{\mathbf{S H}(k)}\left(\operatorname{Spec}(R)_{+}, F / \ell^{v}\right)
$$

where $K$ is viewed as a filtered colimit of smooth finitely generated $k$-subalgebras $R$. With these modifications the proof of Theorem 4.10 goes through. Theorems 4.12-4.16 remain valid when replacing $f_{\sharp}$ by $f^{*}, f^{*}$ by $f_{*}$, and "reflection" by "coreflection." In the last sentence in the proof of 4.13 we should have written "Hom $\operatorname{HH}_{(K)}\left(f^{*} E, f^{*}(L / \ell \wedge F)\right)$ " instead of " $\operatorname{Hom}_{\mathbf{S H}(K)}\left(E, f^{*}(L / \ell \wedge F)\right)$ ".

Another typo appears in Remark 2.4, where " $\mathbf{P}_{+}^{2}$ " has to be replaced with " $\mathbf{P}$ ",

In conclusion, we express our gratitude to Markus Spitzweck for his observation and to Joseph Ayoub for encouragement.

\section{References}

1. Cisinski, D.-C., Déglise, F.: Triangulated categories of mixed motives (preprint, 2009). arXiv:0912. $2110 \mathrm{v} 2$ [math.AG]

2. Röndigs, O., Østvær, P.A.: Rigidity in motivic homotopy theory. Math. Ann. 341(3), 651-675 (2008) 